\title{
Arida A, Fragiadaki K, Giavri E, Sfikakis PP (2010) Anti-TNF Agents for Behçet's disease: analysis of published data on 369 patients. Semin Arthritis Rheum [PMID:21168186]
}

\author{
Nicole Stuebiger • Dicle Hazirolan • Uwe Pleyer
}

Received: 13 May 2011 / Accepted: 16 May 2011 / Published online: 27 July 2011

(C) Springer-Verlag 2011

\author{
UNIVERSITÄTSMEDIZIN BERLIN • \\ CAMPUS BENJAMIN FRANKLIN UND VIRCHOW KLINIKUM
}

Long clinical experience tells us that the number of therapies suggested to treat a specific disease is directly proportional to the lack of success in treating that disease.

This certainly applies to Behçet's disease (BD), which as a systemic disorder involving different organs - is often refractory to therapy. This is particularly true if the eyes are affected by vasculitis of the retina.

The list of treatments seems endless, including alkylators, azathioprine, methotrexate, antibiotics, dapsone, levamisole, calcineurin inhibitors (cyclosporine A, tacrolimus), colchicine, thalidomide, corticosteroids, plasma pheresis, interferon alpha (IFN $\alpha$ ), monoclonal antibody to either CD52 (Campath 1) or the interleukin 2 receptor (Daclizumab) and intravenous immunoglobulin, all of which have been applied for this potentially blinding disease.

N. Stuebiger $\cdot$ D. Hazirolan $\cdot$ U. Pleyer

Department of Ophthalmology,

Campus Benjamin Franklin und Virchow Klinikum,

Charité-Universitätsmedizin Berlin,

Berlin, Germany

N. Stuebiger $(\bowtie)$

Department of Ophthalmology,

Campus Benjamin Franklin,

Charité-Universitätsmedizin Berlin,

Hindenburgdamm 30,

12200 Berlin, Germany

e-mail: nicole.stuebiger@charite.de
Recently, Arida A et al. reviewed in Seminars in Arthritis and Rheumatism the available information for another promising candidate in this long list of interventions: anti-TNFalpha agents.

The authors evaluated published data appearing in Medline/Pubmed through March 2010 concerning efficacy and safety for BD patients with unmet medical needs due to severe disease manifestations. This includes ocular, gastrointestinal, mucocutaneous and arthritic, vascular, and central nervous system involvement. They identified 113 original articles from 20 countries reporting on 369 patients receiving TNF blockers. All of these patients were inadequately controlled or intolerant to other immunosuppressive treatments, including interferon in 20 patients with ocular involvement. Two hundred and eighty-six patients (78\%) suffered from intraocular inflammation and were treated either with infliximab (262 patients), adalimumab (16 patients), or etanercept (ten patients). No articles could be found regarding the use of certolizumab or golimumab.

Response to treatment varied significantly with $60 \%$ in the etanercept, $89 \%$ in the infliximab, and $100 \%$ in the adalimumab group respectively. Improvement was defined as absence or significant reduction of subsequent uveitis relapses. Concerning ocular involvement only for the infliximab group, more detailed information was provided: in total, $65 \%$ of patients revealed complete remission during a follow-up of 28 days to 3 years, with a median of 16 months [patients who were resistant to previous IFN treatment responded in $90 \%$ of cases (18/20 patients)]. 
Long-term remission of ocular inflammation, varying from 6 months to 3 years, was reported in $40 \%$ of patients after discontinuation. The remaining individuals experienced relapses, but the majority responded to re-initiation of treatment. The combination of infliximab with azathioprine and/or cyclosporine A (CsA) and/or methotrexate seemed to be superior to mono therapy for sustained ocular remission in prospective studies (Statistical analysis of available data in the treatment of ocular BD revealed a significant difference over infliximab mono therapy only for the combination with CsA but not those with azathioprine or methotrexate. The authors suggest that this could be due to the limited number of patients).

Two individuals from the infliximab group were switched successfully to adalimumab due to hypersensitivity reaction $(n=1)$ and to avoid delayed infusion reactions $(n=1)$.

The authors analysed the current literature, focusing on therapy with TNF blockers in patients with multiple organ manifestations in BD. Interestingly, most information is limited to case reports and case series with less than five patients (86 out of 113 studies). Only 11 papers, mostly open unmasked prospective or retrospective studies report on ten or more patients (with a maximum of 25 patients). Currently, only one randomised, double-masked, placebocontrolled trial in the therapy evaluation on mucocutaneous manifestations and arthritis is available.

They presented a comparison of the most commonly used three TNF blockers in BD: infliximab, adalimumab and etanercept, as mono therapy and in combination with conventional immune suppressants. Especially in ocular $\mathrm{BD}$, infliximab in combination with immune suppressants seems to be superior to mono therapy. Nineteen patients received two different anti-TNF agents, one patient received all three - the information in which group, and why more than one anti-TNF agent was used, is mostly lacking. Even the follow-up times, the definition of remission, the dosages of the additional corticosteroid treatment, the forms of uveitis the BD patients were suffering from, and especially the values of the BCVA (best-corrected visual acuity), are not clearly defined by the authors. In addition, we are wondering why the response and the remission rates in the discussed studies are not scored by using an activity index, such as the "uveitis scoring system" described by BenEzra et al. [1] and for the assessment of disease activity in general, e.g,. the "BD current activity form (BDCAF)".

Focusing on the ocular involvement, the authors attenuate differences between the TNF blocker and IFN therapy. First of all, they describe 20 patients with ocular BD who did not respond to IFN $\alpha$ therapy, but of whom $90 \%$ did respond to TNF blocker treatment. Unfortunately, they did not mention which dosage of IFN and which dosage of additional corticosteroids (or concomitant immune suppressants) these patients received. A closer look at a cited reference of Accorinti et al. [2] discloses that patients without remission during IFN treatment received 3 Mio IU IFN $\alpha$ three times weekly only. This would have been an interesting information. Referring to a review published by Kötter et al. in 2004 [3], summarizing current practices in IFN therapy for $\mathrm{BD}$, revealed that long-term remissions of ocular disease are more likely achieved applying higher initial doses of IFN (e.g., 6 Mio IU/day). It also has been postulated that the effect of IFN could be antagonized by high doses of corticosteroids or immune suppressants, or by anti-IFNantibodies, information that is unfortunately lacking in the current review.

With regard to long-term remission, there is a remarkable difference to emphasize when comparing the two classes of biologics. Whereas the response rate using either IFN $(88 \%$ to $98 \%$ ) [4-9] or infliximab (89\%) is comparable, longterm remission differs: after discontinuation of infliximab treatment, $40 \%$ of patients achieved long-term remission of ocular inflammation varying from 6 months to 3 years. In comparison, $57 \%$ to $68 \%$ of the IFN-treated (high-dose regimen) patients could discontinue their treatment and were free of relapses during a follow-up period of 1.5 to 13 years $[4,5]$.

In addition, time to response of ocular manifestations to antiTNF treatment ranged from 2 weeks with regard to healing of retinal vasculitis to 3 weeks with regard to disappearance of macular edema (mean 11 days). For IFN-treated patients, regressing and disappearance of retinal vasculitis and macular edema is described as being in between 2 and 4 weeks; overall, the time to response (response $=$ score $<50 \%$ of initial value) is reported for the Posterior Uveitis Score with a median of 3 weeks $[3,4,6,8]$.

Reported serious adverse effects of TNF alpha-blocking drugs include increased risk of malignancy, tuberculosis, multiple sclerosis, optic neuritis, congestive heart failure, and lupus-like reactions. TNF alpha blockers are contraindicated in patients with a history of these conditions. In the majority of patients, side effects are mild, either in short-term or in long-term administration, and consisted mainly of infusion/hypersensitivity reactions (local erythema at injection site, atopic dermatitis, flushing, hypertension, chest discomfort, rash, fatigue, fever), not requiring withdrawal of therapy.

Common side effects due to IFN alpha therapy are influenza-like syndrome (fever, chills, headache, myalgia, and fatigue), especially in the beginning of treatment. Other reversible and treatable adverse effects include leucopenia, 
thrombocytopenia, nausea, vomiting, diarrhoea, hair loss, weight loss, dermatitis, increase in liver enzyme levels, worsening of psoriasis, epileptic seizures, ulcerations at the site of injection, and depression.

Combined adverse effects of both drugs are the induction and formation of various autoantibodies (e.g. ANA, anti-dsDNA), mostly clinically insignificant. In addition, the appearance of neutralising antibodies either to TNF blocking agents [in the case of infliximab: human antichimeric antibody (HACA)/antibodies-to-infliximab (ATI)] or to IFN alpha may occur. Measurement of these antibodies in the serum of patients would provide direct information on this issue.

Therefore, concomitant use of other immunosuppressive agents together with the TNF alpha blocker has been suggested to prevent the development of antibody formation - but not in case of IFN treatment, due to possible antagonistic effects between the interferons and the immunosuppressives.

In essence, biologics such as TNF blockers seem to be an effective therapy in patients affected with $\mathrm{BD}$, especially in those with ocular disease. With regard to the rate and time to achieve remission, TNF blocking agents and IFN appear to be equally effective. However, to obtain the important goal of long-term remission, the current literature favours IFN over TNF blockers with regard to the number of patients and the duration of remission. Interestingly enough, patients who failed to respond to low-dose IFN therapy responded well to TNF blockers, indicating that several therapeutic pathways may exist.

In 2008, the EULAR (European League against Rheumatism) published recommendations for the therapy of BD. They suggest in ocular BD: "If the patient has severe eye disease defined as $>2$ lines drop of visual acuity on a 10/10 scale, and/or retinal disease (retinal vasculitis or macular involvement), it is recommended that either cyclosporine A or infliximab be used in combination with azathioprine and corticosteroids; alternatively, interferon alpha with or without corticosteroids could be used instead" [9].
Unfortunately, both drugs are up to now off-label therapies in most countries, exept Japan. Therefore randomised studies against an on-label immunosuppressant are needful - for alpha IFN a German national comparative multicenter trial in ocular BD versus CsA ("INCYTOB" www.clinicaltrials.gov) is currently ongoing.

\section{References}

1. BenEzra D, Cohen E (1986) Treatment and visual prognosis in Behçet's disease. Br J Ophthalmol 70:589-592

2. Accorinti M, Pirraglia MP, Paroli MP, Priori R, Conti F, PivettiPezzi P (2007) Infliximab treatment for ocular and extraocular manifestations of Behçet's disease. Jpn J Ophthalmol 51:191196

3. Kötter I, Günaydin I, Zierhut M, Stübiger N (2004) The use of interferon alpha in Behçet's disease: review of the literature. Semin Arthritis Rheum 33:320-335

4. Deuter CM, Zierhut M, Möhle A, Vonthein R, Stübiger N, Kötter I (2010) Long term remission after cessation of interferon- $\alpha$ treatment in patients with severe uveitis due to Behçet's disease. Arthritis Rheum 62:2796-2805

5. Geudry J, Wechsler B, Terrada C, Gendron G, Cassoux N, Fardeau C, Lehoang P, Piette JC, Bodaghi B (2008) Long-term efficacy and safety of low-dose interferon $\alpha 2$ a therapy associated with Behçet's disease. Am J Ophthalmol 146:837-844

6. Kötter I, Zierhut M, Eckstein AK, Vonthein R, Ness T, Günaydin I, Grimbacher B, Blaschke S, Mayer-Riemann W, Peter HH, Stübiger $N$ (2003) Human recombinant interferon- $\alpha 2$ a for the treatment of Behçet's disease with sight threatening posterior or panuveitis. Br J Ophthalmol 87:423-431

7. Krause L, Altenburg A, Pleyer U, Köhler AK, Zouboulis CC, Foerster MH (2008) Long-term visual prognosis of patients with ocular Adamantiades-Behçet's disease treated with interferon- $\alpha 2 \mathrm{a}$. J Rheumatol 35:896-903

8. Stübiger N, Kötter I, Deuter C, Zierhut M (2001) Behçet's disease: uveitis therapy with interferon- $\alpha 2 \mathrm{a}$ - prospective study in 33 patients. Klin Monatsbl Augenheilkd 218:768-773

9. Hatemi G, Bang D, Bodaghi B, Chamberlain AM, Gul A, Houman MH, Kötter I, Olivieri I, Salvarani C, Sfikakis PP, Siva A, Stanford MR, Stübiger N, Yurdakul S, Yazici H: EULAR Expert Committee (2008) EULAR recommendations for the management of Behçet's disease. Ann Rheum Dis 67:16561662 